\title{
Das Deutsche Zentrum für Lungenforschung - Translationale Forschung für Prävention, Diagnose und Therapie von Atemwegserkrankungen
}

\author{
The German Centre for Lung Research - Translational Research for the \\ Prevention, Diagnosis and Treatment of Respiratory Diseases
}

Autoren

Institute
W. Seeger ${ }^{1}$, T. Welte ${ }^{2}$, O. Eickelberg ${ }^{3}$, M. Mall ${ }^{4}$, K. F. Rabe ${ }^{5}$, B. Keller ${ }^{6}$, S. Winkler ${ }^{6}$, U. Koller ${ }^{6}$

Die Institutsangaben sind am Ende des Beitrags gelistet. eingegangen $\quad 3.7 .2012$

akzeptiert 3.7.2012

Bibliografie

DOI http://dx.doi.org/

10.1055/s-0032-1310086

Pneumologie 2012; 66: 464-469

(c) Georg Thieme Verlag KG

Stuttgart · New York

ISSN 0934-8387

Korrespondenzadresse

Prof. Dr. med. Tobias Welte

Klinik für Pneumologie

Medizinische Hochschule

Hannover

Carl-Neuberg-Straße 1

30625 Hannover

welte.tobias@mh-hannover.de

\section{Zusammenfassung}

$\nabla$

Lungenerkrankungen zählen in Deutschland und weltweit zu den häufigsten Todesursachen und Erkrankungen mit erheblichen Kosten für die Gesundheitssysteme. Bislang gibt es für die meisten chronischen Lungenerkrankungen kaum wirksame Therapieansätze. Das Bundesministerium für Bildung und Forschung hat daher nach dem Vorbild der amerikanischen National Institutes of Health die Gründung eines Deutsches Zentrums für Lungenforschung (DZL) unterstützt, damit zügig neue Optionen in Diagnose, Therapie und Prävention von Lungenerkrankungen entwickelt werden können. Dem Deutschen Zentrum für Lungenforschung gehören die führenden universitären und außeruniversitären Einrichtungen für Lungenforschung in Deutschland an. Um den Transfer wissenschaftlicher Erkenntnisse aus der Grundlagenforschung in klinische Lösungen für eine bessere Patientenversorgung zu ermöglichen, werden im DZL grundlagen-, krankheitsund patientenorientiert Forschungsansätze miteinander verbunden. Das DZL ist eines der sechs Deutschen Zentren für Gesundheitsforschung (Neurologische Erkrankungen, Diabetes, Herzund Kreislauferkrankungen, Infektionskrankheiten, Krebserkrankungen und Lungenerkrankungen), mit denen neue Forschungsstrukturen für optimierte Translationsprozesse zur Bekämpfung der großen Volkskrankheiten geschaffen wurden.

\section{Einleitung}

$\nabla$

Der 9. Juni 2011 war für die Gesundheitsforschung in Deutschland ein entscheidender Tag: Bundesforschungsministerin Prof. Annette Schavan eröffnete in Berlin offiziell die sechs Deutschen Zentren für Gesundheitsforschung, an denen künftig die besten Wissenschaftlerinnen und Wissenschaftler unter einem Dach große Volks-

\section{Abstract \\ $\nabla$}

Respiratory diseases are one of the most important causes of mortality with tremendous costs for health care systems, not only in Germany, but worldwide. Up to now treatment options for most of these chronic diseases are limited. The German Ministry for Research and Education (BMBF) - following the example of the US National Institute of Health have supported the foundation of a German Centre for Lung Research (DZL) to speed up the development of preventive, diagnostic and therapeutic measures. Not only universities, but also non-university based research institutes are part of the DZL. To allow the translation from basic research experience into clinical practice to improve patient care, basic research orientated approaches will be combined with disease and patient focused approaches. The DZL is one of six German Centres for Health Care Research (neurological diseases, diabetes, cardiovascular diseases, infectious diseases, cancer, and lung diseases) for the optimisation of translational processes to overcome the burden of major diseases.

krankheiten wie Krebs, Herz-Kreislauf-, Stoffwechsel-, Infektions-, Lungen- und neurodegenerative Erkrankungen erforschen und Ergebnisse für Prävention und Therapie schneller in die Anwendung bringen werden.

Das Deutsche Zentrum für Lungenforschung (DZL) ist eines dieser sechs Zentren. Insgesamt 18 universitäre und außeruniversitäre Einrichtungen an fünf Standorten werden im DZL die Erfor- 


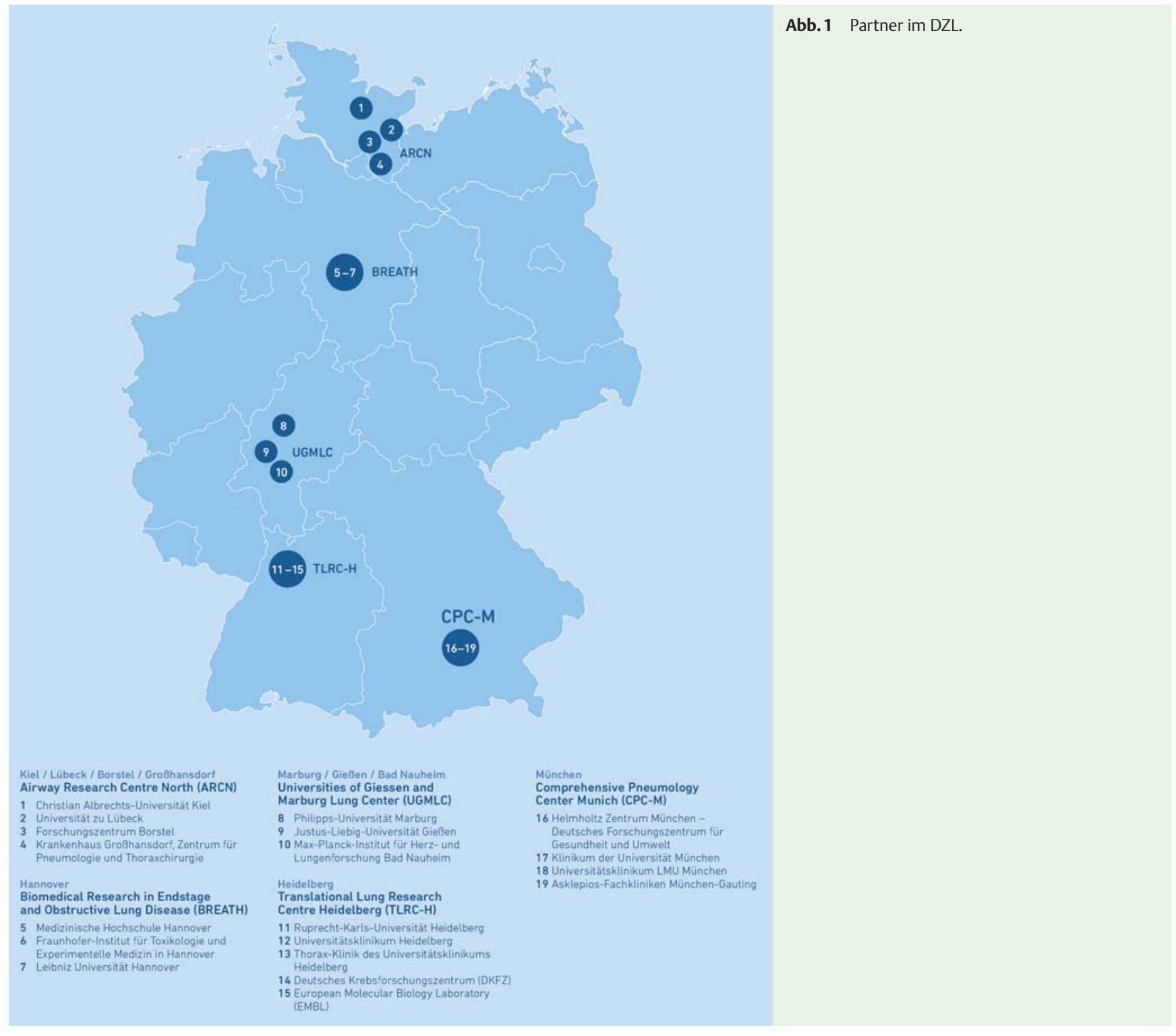

schung von Lungenerkrankungen weiter voran bringen. Die Notwendigkeit eines solchen Zentrums unterstreichen zahlreiche Fakten:

Die Weltgesundheitsorganisation WHO listet unter den zehn weltweit häufigsten Todesursachen vier Lungenerkrankungen auf. Jeder fünfte Todesfall geht heute auf eine Lungenerkrankung oder ihre Folgeerkrankungen zurück. Bis 2030 wird ein weiterer starker Anstieg der Fallzahlen prognostiziert. Die meisten schweren Lungenerkrankungen sind bis heute nicht heilbar und können nur symptomatisch behandelt werden, was mit einem hohen volkswirtschaftlichen Aufwand einhergeht. Mit den Fallzahlen werden auch die prognostizierten Kosten für die Behandlung von Lungenleiden in den nächsten Jahrzehnten noch weiter steigen. Studien zu den Ursachen der schweren Krankheitsbilder und neuen diagnostischen, therapeutischen und präventiven Optionen sind daher dringend notwendig.

\section{Ziele des Deutschen Zentrums für Lungenforschung}

Die Wissenschaftler und Kliniker im DZL erforschen gemeinsam neue Optionen für Vorsorge, Diagnose und Therapie von Lungenerkrankungen. Die Partner vertreten große universitäre und außeruniversitäre Forschungseinrichtungen, darunter Universitätskliniken, Helmholtz-Zentren, Max-Planck-, Leibniz- und Fraunhofer-Institute, das European Molecular Biology Laboratory (EMBL) sowie verschiedene renommierte Lungenfachzentren. Sie bilden Verbünde an fünf Standorten: ARCN (Borstel/Lübeck/ Kiel/Großhansdorf), UGMLC (Gießen/Marburg/Bad Nauheim) sowie BREATH (Hannover), TLRC-H (Heidelberg) und CPC-M (München/Neuherberg/Gauting) ( Abb.1). Unter den Mitgliedern befinden sich Universitätskliniken und Lungenfachkliniken mit mehr als 1000 Betten für Patienten mit Lungenerkrankungen, die den direkten Zugang zum Patienten ermöglichen.

In einzelnen Bereichen und für bestimmte Projekte ist die Mitarbeit von nicht im DZL verankerten Forschungsstandorten erwünscht und jederzeit möglich. 


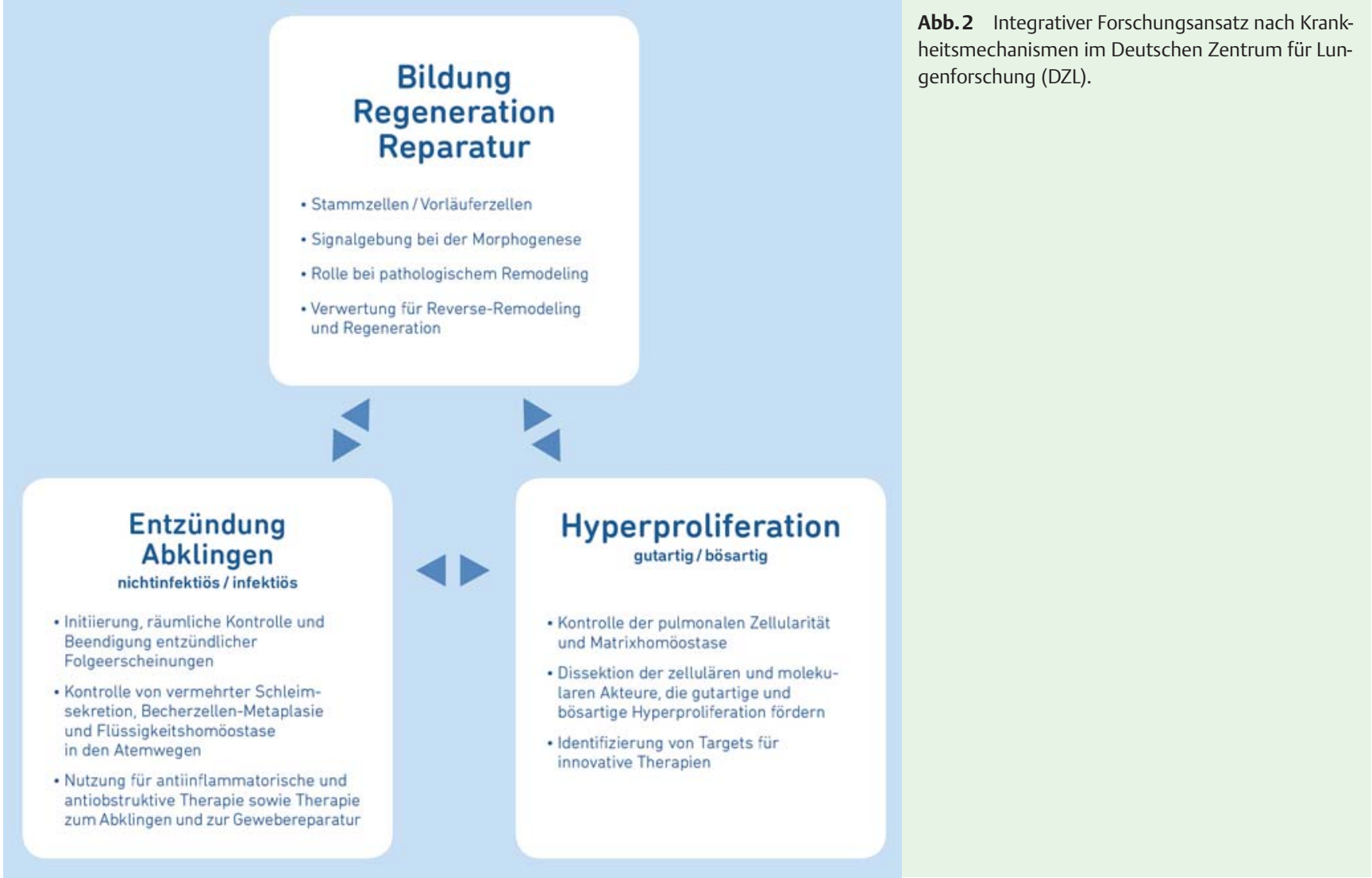

Vom Labor zum Krankenbett - Ein translationaler Ansatz Erkenntnisse aus der Grundlagenforschung unmittelbar für die Diagnostik und Therapie von Krankheiten anwenden und umgekehrt Ergebnisse aus Patientenstudien ins Labor zurückfließen lassen-das ist der Kerngedanke translationaler Forschung in der Medizin. Diesen Brückenschlag vollzieht das Deutsche Zentrum für Lungenforschung. Ausgehend von molekularen Ansatzpunkten für Krankheitsmechanismen bis hin zu klinischen Studien in definierten Krankheitskohorten und einem patientenorientierten Gesundheitsmanagement deckt das DZL die gesamte Wertschöpfungskette $a b$. Möglich wird dies erst durch die große Vielfalt an Expertise, darunter Molekularbiologen, Entwicklungsbiologen, experimentelle Lungenforscher, Pneumologen und Intensivmediziner, Epidemiologen und Gesundheitsökonomen.

\section{Gemeinsame Krankheitsmechanismen im Visier}

Die Krankheitsmechanismen aller schweren Lungenerkrankungen haben eine Gemeinsamkeit, der Wissenschaftler und Kliniker im DZL größte Aufmerksamkeit widmen: Sie unterliegen einem dynamischen Zusammenspiel von drei Prozessen in der Lunge: Regenerations- und Reparaturmechanismen, infektiösem oder nichtinfektiösem Entzündungsgeschehen sowie gut- und bösartigem Zell- bzw. Gewebewachstum. ( $\bullet$ Abb.2)

\section{Aufbau und Struktur des deutschen Zentrums für Lungenforschung}

Das DZL unterliegt einer sehr gliedrigen Struktur mit Krankheitsfeldern und Plattformen, einem Lenkungsausschuss, dem Vorstand und einem internationalen wissenschaftlichen Beirat. ( Abb.3)
In den Krankheitsfeldern und Plattformen werden gemeinsame wissenschaftliche Projekte vorangetrieben, laufend evaluiert und bei Bedarf angepasst. Auf dieser Ebene erfolgt auch die Festsetzung von Prioritäten zwischen den einzelnen Projekten eines jeden Feldes. Der Lenkungsausschuss evaluiert den Fortschritt des gesamten DZL und koordiniert die verschiedenen Krankheitsfelder und Plattformen. Eine zentrale Rolle für kontinuierliches Management und strategische Entscheidungen kommt dem Vorstand des DZL zu. Der internationale wissenschaftliche Beirat berät das DZL und passt bei Bedarf das Gesamtkonzept neuen Gegebenheiten an.

\section{Rechtsform}

In seiner Rechtsform ist das DZL ein gemeinnütziger Verein, Vereinssitz ist Gießen, Vorsitzender des Vereins und zugleich Sprecher des DZL ist Werner Seeger, wissenschaftlicher Koordinator des Lungenzentrums der Universitäten Gießen und Marburg (UGMLC). Weitere Mitglieder des Vorstands und stellvertretende Vorsitzende sind Oliver Eickelberg (Koordinator CPC-M), Marcus Mall (Koordinator TLRC-H), Klaus F. Rabe (Koordinator ARCN) und Tobias Welte (Koordinator BREATH). Vereinszweck ist die Förderung von Wissenschaft und Forschung im Bereich der Lungenerkrankungen durch Einrichtung eines Forschungsverbunds, der interdisziplinäre Forschung und klinische Anbindung in einem ganzheitlichen Ansatz vereint.

\section{Mittelverwaltung}

Das Helmholtz Zentrum München, eine der DZL-Partner-Einrichtungen, koordiniert den administrativen Implementierungsprozess. Hierzu wurde in München ein administratives Fördermittelmanagement aufgebaut, um die ordnungsgemäße und effiziente Beantragung, Bewirtschaftung und Abwicklung der 


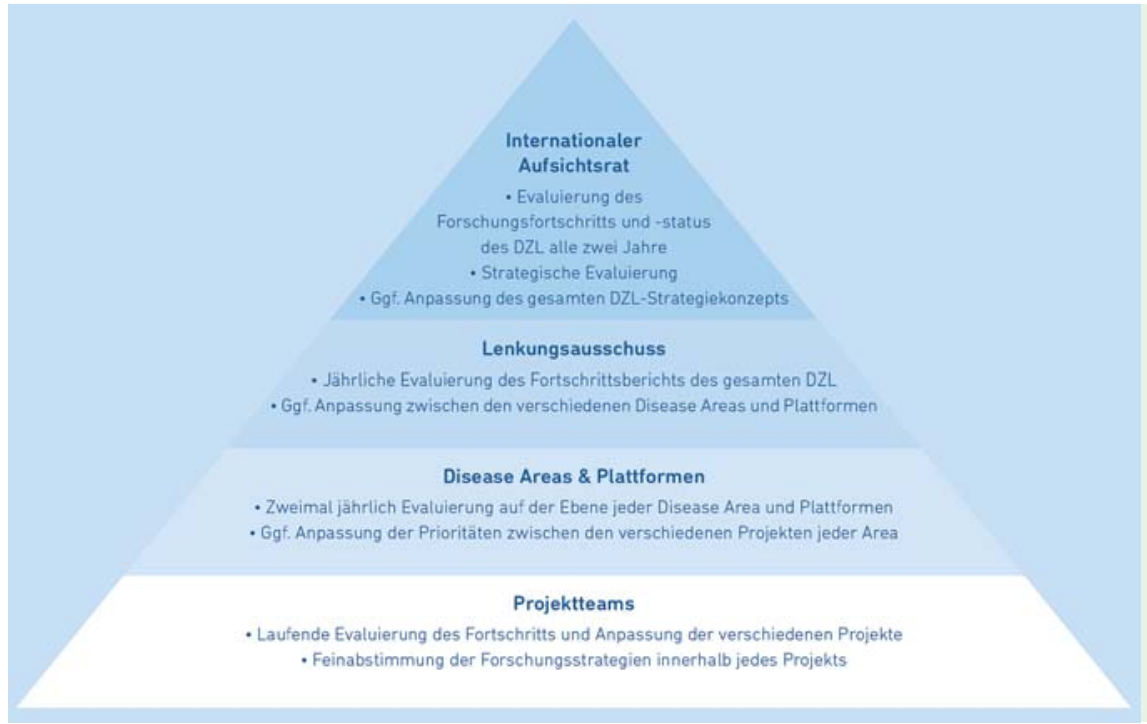

Abb.3 Administrative Struktur des Deutschen Zentrums für Lungenforschung.

\begin{tabular}{|c|c|c|}
\hline & Fokus & \\
\hline $\begin{array}{l}\text { Klinische } \\
\text { Studien }\end{array}$ & $\begin{array}{l}\text { - Neuartige therapeutische Targets } \\
\text { - Patientenbezogene Ergebnisse } \\
\text { - Gesundheitsökonomie }\end{array}$ & Therapie \\
\hline Kohorten & $\begin{array}{l}\text { - Beziehung Struktur - Funktion } \\
\text { - Aktivität } \\
\text { - Innovative diagnostische Ansätze } \\
\text { - Biomarker } \\
\text { - Phänoptypisierung }\end{array}$ & Diagnostik \\
\hline $\begin{array}{l}\text { Modelle } \\
\text { Zellkulturen } \\
\text { Tiermodelle }\end{array}$ & $\begin{array}{l}\text { - Genetische / epigenetische Regulierung } \\
\text { - Strukturelle Lungenzellen } \\
\text { - Neuronen } \\
\text { - Angeborenes / adaptives Immunsystem } \\
\text { - Remodeling } \\
\text { - Reparatur / Regeneration }\end{array}$ & Mechanismen \\
\hline
\end{tabular}

Abb.4 DZL-Forschungsansatz bei COPD.

wissenschaftlichen Projekte zu gewährleisten. Entsprechend des Helmholtz-Finanzierungsschlüssels kommen 90 Prozent der Fördermittel für das DZL vom Bund und die übrigen 10 Prozent aus dem Bundesland des jeweiligen DZL-Standortes.

\section{Disease Areas und Plattformen - die Grundpfeiler des DZL}

Zwei Säulen bestimmen die Grundstruktur des DZL: Forschungsverbünde mit Fokus auf die wesentlichen Krankheitsbilder sowie Plattformen, die von allen Partnern gemeinsam genutzt werden.

\section{Von Asthma bis Lungenkrebs - Disease Areas im Fokus des DZL}

Die langfristige Vision des DZL ist klar: Es will mit seiner Forschung substanziell dazu beitragen, Diagnostik, Therapie und Prävention von schweren Lungenerkrankungen zu verbessern. Das DZL befasst sich vornehmlich mit den Krankheitsbildern, die heute noch als nicht heilbar gelten und verfolgt dabei konkrete Ziele für die einzelnen Indikationen. Abb.4 zeigt am Beispiel der COPD, wie Erkenntnisse aus der Grundlagenforschung in die Entwicklung von Biomarkern für eine verbesserte Phänotypisie- rung von Patienten eingehen sollen und wie dies wiederum die Entwicklung neuer Therapien beeinflusst.

Dazu gehören Asthma und Allergien, die chronisch-obstruktive Lungenerkrankung (COPD), Mukoviszidose, akute Lungenschädigungen und Lungenversagen, diffuse parenchymatöse Lungenleiden sowie Lungenhochdruck, schwere Lungenerkrankungen im Endstadium und Lungenkrebs.

\section{Das Handwerkszeug des DZL - Moderne Forschungs- plattformen}

Eine wichtige Voraussetzung für erfolgreiche Forschung sind leistungsstarke und hochmoderne Technologien und Forschungsplattformen, die von allen Partnern gemeinsam genutzt werden können. Die im DZL vereinten Einrichtungen verfügen bereits heute über eine Vielzahl solcher Einheiten: Dazu zählen Stateof-the-art-Technologien zur Phänotypisierung, hochmoderne und leistungsfähige Hochdurchsatztechnologien, Genomik- und Proteomics-Einheiten oder Biobanken.

Aufbauend auf vorhandenen Strukturen in den Partnereinrichtungen wird das DZL für drei zentrale Bereiche neue DZL-weite Plattformen einrichten: eine zentrale Biobank, ein Bord für klinische Studien sowie eine Bildgebungs-Plattform. 
Zentrale DZL-Biobanking-Plattform. Mit einer zentral organisierten DZL-Biobanking-Plattform können künftig alle Mitglieder des DZL, aber auch externe Partner, einfachen und direkten Zugang zu Probenmaterial von Patienten erlangen, die an einem der im DZL behandelten Krankheitsbilder leiden. Bestehende Materialsammlungen werden online vernetzt und bieten Informationen über Art und Standort des vorhandenen Probenmaterials. Die Plattform baut auf bestehende Biobanken in Heidelberg, Hannover, München und Gießen auf:

- die Lungen-Biobank in Heidelberg (Gewebeproben von über 2.500 vornehmlich Lungenkrebspatienten, Serum- und Plasmaproben von über 5.000 Patienten),

- die Lungen-Biobank in Hannover mit unter anderem DNA-/ Plasma- und Serumproben von Asthma- und Allergiepatienten und Patienten nach Lungentransplantation,

- Bioprobenbanken in München/Neuherberg mit Material von Transplantationen, Biopsien sowie DNA-/Plasma- und Serumproben aus großen epidemiologischen Bevölkerungskohorten wie KORA mit rund 200000 Studienteilnehmern und

- die Biobank in Gießen u.a. mit über 3000 Proben von Patienten mit Lungenhochdruck und Lungenfibrose.

Vor dem Hintergrund der Harmonisierung europäischer Biobanken werden die Probensammlungen im DZL als Teil der BBMRI-Initiative (Biobanking and Biomolecular Resources Research Infrastructure) gehandhabt. Für das Daten- und Qualitätsmanagement sowie infrastrukturelle Fragen wird das DZL zudem mit der Technologie- und Methodenplattform für die vernetzte medizinische Forschung (TMF) zusammenarbeiten.

\section{Nachwuchsförderung auf hohem Niveau - Die DZL-School \\ $\nabla$}

Über 250 Doktoranden und Postdoc-Wissenschaftler verschiedener Fachrichtungen arbeiten in den Forschungsprojekten der fünf DZL-Standorte. Ihnen soll künftig die breite Expertise der DZLWissenschaftler aller Standorte und aller Aspekte der Erforschung von Lungenerkrankungen zugutekommen. Die DZLSchool, geplant als enge Kooperation mit INSERM-basierter Lungenforschung in Frankreich als deutsch-französische Lungenschule, wird exzellente Ausbildung fördern und damit den weiteren erfolgreichen beruflichen Werdegang der Doktoranden sichern und optimieren. Im Rahmen eines standortübergreifenden Ausbildungsprogramms werden die Doktoranden und Nachwuchswissenschaftler an Methodenworkshops, wissenschaftlichen Symposien sowie karrierefördernden Maßnahmen teilnehmen. Dies fördert den persönlichen Austausch zwischen Studenten an den fünf DZL-Standorten sowie mit französischen Lungenforschungseinrichtungen.

Über die reine wissenschaftliche Ausbildung hinaus erwarten sich die Koordinatoren aus der DZL-School zusätzlich einen Corporate Identity stiftenden Effekt, der für das junge Deutsche Zentrum für Lungenforschung auf lange Sicht Früchte tragen wird.

\section{Wissen über Lungenerkrankungen verbreiten - der Lungeninformationsdienst \\ $\nabla$}

Umfassendes und fundiertes Wissen über schwere Lungenerkrankungen steht Patienten bislang nur teilweise und oft in wenig aufbereiteter Form zur Verfügung. Es fehlt in vielen Bereichen aktuelle, neutrale, qualitätsgesicherte und vor allem gebündelte Information. Mit einem umfassenden Informationsangebot bietet der Lungeninformationsdienst Patienten und deren Angehörigen Wissen rund um Lungenerkrankungen aus Forschung und Medizin, das allgemeinverständlich aufbereitet ist. Die Nähe des Dienstes zur Wissenschaft gewährleistet ein Maximum an Seriosität, Aktualität und Neutralität.

Damit leistet der Dienst einen entscheidenden Beitrag, um eine Brücke von der Forschung zum direkten Nutzen für den Patienten zu schlagen. Zugleich wird der Lungeninformationsdienst die Wahrnehmung für Lungenerkrankungen in der Bevölkerung schärfen. Er kann zudem dazu beitragen, den Stellenwert der Lungenforschung in der gesamten Forschungslandschaft zu verbessern. Der Lungeninformationsdienst ist am Helmholtz Zentrum München angebunden.

In einer ersten Phase bietet der Lungeninformationsdienst eine Online-Plattform mit aktuellem Wissen über Diagnostik, Therapie, Prävention und aktuelle Forschungsansätze zu Lungenerkrankungen ( Abb.5). Patientenorientierte Service-Informationen über Kliniken, Fachverbände, Selbsthilfeorganisationen sowie Veranstaltungs- und Literaturhinweise ergänzen das Angebot. Ein monatlich erscheinender Newsletter informiert aktiv über neue Inhalte des Lungeninformationsdienstes.

Mit einem Runden Tisch für Patientenorganisationen und regelmäßigen Expertenforen fördert der Lungeninformationsdienst zudem den direkten Austausch zwischen Experten und Patienten. Im Laufe des Jahres 2012 wird eine Patienten-Hotline aufgebaut, in der medizinisches und psychologisches Fachpersonal persönliche Anfragen von Patienten und deren Angehörigen zu Lungenerkrankungen beantwortet.

\section{Synergien nutzen - Kooperationen mit Dritten \\ $\nabla$}

Über die Kooperationen innerhalb des DZL und zwischen den Forschungsprogrammen der DZL-Partner hinaus erhoffen sich die Beteiligten signifikante Synergien und künftige Kooperationen mit anderen deutschen Zentren der Gesundheitsforschung wie etwa dem Deutschen Zentrum für Infektionsforschung, dem Deutschen Konsortium für translationale Krebsforschung sowie dem Deutschen Zentrum für Herz-Kreislauf-Forschung. Strategische Partnerschaften mit Forschungseinrichtungen im weiteren Umfeld sollen dazu führen, dass die Forschung im DZL zügig zu konkretem Nutzen für die Gesellschaft führt. Zudem ist eine enge Kooperation mit weiteren Lungenforschungsnetzwerken wie CAPNETZ und COSYCONET vorgesehen: Beide Einrichtungen sollen in den kommenden Jahren an das DZL assoziiert werden. Das DZL bietet zudem eine Vielfalt an Möglichkeiten für wissenschaftliche Kooperationen mit Nicht-DZL-Partnern, etwa im Rahmen wissenschaftlicher Projekte. Dabei können Daten und Materialien aus DZL-Plattformen auf Antrag auch Nicht-DZL-Partnern zur Verfügung gestellt sowie Nachwuchswissenschaftlerinnen und -wissenschaftler in DZL-Projekte eingebunden werden. 


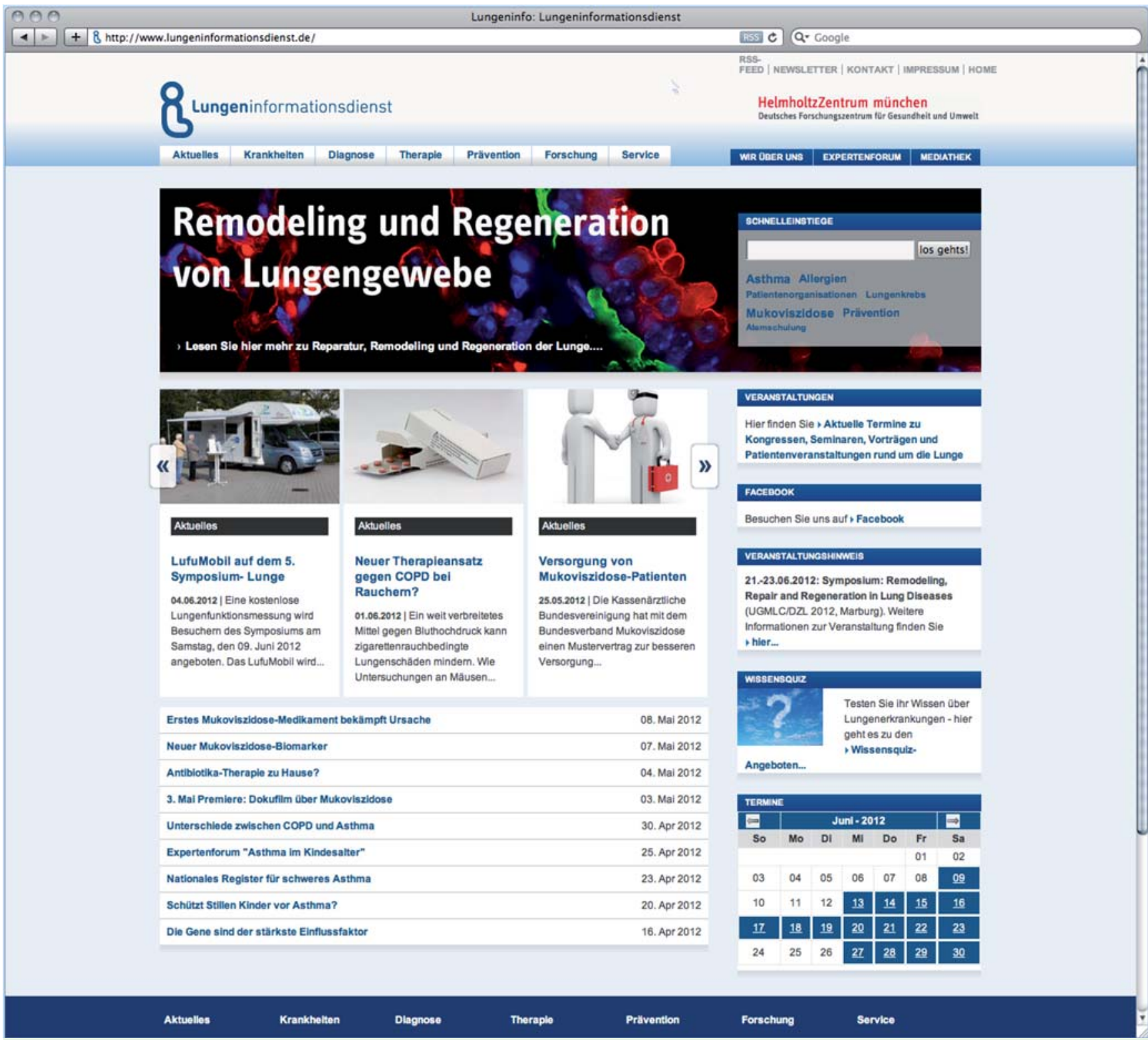

Abb.5 Online-Angebot des Lungeninformationsdienstes (www.lungeninformationsdienst.de.)

Weitere wertvolle Partner für das DZL sind Organe wie die Zeitschrift Pneumologie, mit deren Unterstützung das DZL seine Projekte, Forschungsergebnisse und Termine an das interessierte Fachpublikum kommunizieren kann.

\section{DZL - Veranstaltungen \\ $\nabla$}

2012 wird das DZL zum ersten Mal und dann jährlich wiederkehrend an einem seiner Standorte eine wissenschaftliche Tagung abhalten. Erstmals wurde diese vom 21. bis 23. Juni vom Universities of Gießen and Marburg Lung Center (UGMLC) in Marburg ausgerichtet. Themenschwerpunkt der diesjährigen Tagung war „Remodeling, Repair and Regeneration in Lung Diseases“. Die Jahrestagung 2013 wird in München stattfinden.

\section{Interessenkonflikt}

$\nabla$

Die Autoren geben an, dass kein Interessenkonflikt besteht.

Institute

${ }^{1}$ Universities of Gießen and Marburg Lung Center (UGMLC), Mitglied im DZL

${ }^{2}$ Breath (Biomedical Research in Endstage and Obstructive Lung Disease

Hannover), Mitglied im DZL

${ }^{3}$ Comprehensive Pneumology Center, Klinikum der Ludwig-Maximilians-

Universität München und Helmholtz Zentrum München, Mitglied im DZL

${ }^{4}$ Heidelberg Translational Lung Research Center (TLRC), Mitglied im DZL

${ }^{5}$ Airway Research Center North, Mitglied im DZL

${ }^{6}$ Helmholtz Zentrum München, Abteilung Kommunikation, Mitglied im DZL 